The Journal of Animal \& Plant Sciences, 31(2): 2021, Page: 441-449

ISSN (print): 1018-7081; ISSN (online): 2309-8694

\title{
EFFECTS OF DETOMIDINE AND MIDAZOLAM ADMINISTERED ALONE OR COMBINED, ON INDUCTION DOSE AND QUALITY OF PROPOFOL AND KETAMINE ANESTHESIA IN GOATS
}

\author{
M. Asif ${ }^{1}$, M. A. Khan ${ }^{1,3}$, H. Akbar ${ }^{1}$ and M. O. Omer ${ }^{2}$ \\ ${ }^{1}$ Department of Clinical Medicine \& Surgery, Faculty of Veterinary Sciences, ${ }^{2}$ Department of Pharmacology \& \\ Toxicology, Faculty of Bio-Sciences, University of Veterinary \& Animal Sciences, Lahore, Pakistan, 54000. ${ }^{3}$ Riphah \\ International University, Riphah College of Veterinary Sciences, Lahore, Pakistan, 54000. \\ Corresponding Author's Email: muhammad.asif@uvas.edu.pk
}

\begin{abstract}
Pre-anesthetic treatment directly affects dose of induction agents that may result in least complication due to low anesthesia intake. The study was designed to investigate the effects of detomidine and midazolam on induction dose and quality of propofol and ketamine anesthesia in goats. Twelve female goats were randomly divided into six groups comprising two animals each. Control group (A) received (Normal saline+ propofol @ $4.20 \pm 0.10 \mathrm{mg} / \mathrm{kg}$ ). While, group B received (detomidine@ $15 \mu \mathrm{g} / \mathrm{kg}+$ propofol), C received (detomidine@ $15 \mu \mathrm{g} / \mathrm{kg}+$ ketamine), D received (midazolam@0.25mg/kg+ propofol), E received (midazolam @ $0.25 \mathrm{mg} / \mathrm{kg}+$ ketamine) and group F received combination of all (detomidine @7 $7 \mu \mathrm{g} / \mathrm{kg}+$ midazolam @ $0.12 \mathrm{mg} / \mathrm{kg}+$ propofol+ ketamine). The degree of sedation and analgesia, induction dose, \% age reduction in induction dose, anesthesia quality and clinico-physiological parameters were evaluated. Detomidine-midazolam when administered combined, followed by induction with combination of propofol-ketamine caused deep sedation and analgesia, maximum \% age reduction in induction dose and increased duration of anesthesia that differed significantly $(\mathrm{p} \leq 0.05)$ from control and other treatment groups. Detomidine and midazolam when administered alone, followed by induction with propofol and ketamine caused mild sedation and analgesia, marked \% age reduction in induction dose and increased duration of anesthesia that differed non significantly $(p>0.05)$ from each other. The significant $(p \leq 0.05)$ changes in heart rate \& respiratory rate and non-significant $(p>0.05)$ changes in rectal temperature were noticed in all groups. It is concluded that the combination of detomidine-midazolampropofol-ketamine was found to be safe and economical for short surgical procedures in goat.
\end{abstract}

Keywords: Detomidine, midazolam, sedatives, propofol, ketamine, anesthetics, goat. https://doi.org/10.36899/JAPS.2021.2.0233

Published online October 03,2020

\section{INTRODUCTION}

Surgical management of animals needed safe pre-anesthetics and ideal anesthetic which produced deep sleep, analgesia, amnesia, and muscle relaxation (Zeedan et al., 2014). The concept of pre-anesthetic medication before the induction of anesthetic agents is well acknowledged in veterinary practice. A suitable preanesthetic treatment may help in cardiovascular stability, good sedation \& analgesia and better recovery from anesthesia (Waelbers et al., 2009). Pre-anesthetic treatment directly affects dose of anesthetic agents that may result in least complication due to low anesthesia intake (Bednarski et al., 2011). Pre-anesthetic treatment have also been recommended in goats before administering the induction agents (Galatos, 2011). Different sedatives (xylazine, detomidine, dexmedetomidine, daizepam, midazolam and butorphenol) are being used now a days to restraint the animals as a pre-anesthetic agents (Brighton, 2013; Shah et al., 2013). Detomidine produced dose dependent sedation and analgesia in ruminants (Tunio et al., 2016;
EL-Kammar et al., 2014). Detomidine was found better option due to its good sedative, strong analgesic and less cardiorespiratory effects than xylazine (Hall et al., 2001). Pre-anesthetic treatment with midazolam have been found to cause reduction in the amount of inhalant or injectable anesthetic agents both in man and different animal species (Hendrickx et al., 2008; Dzikiti et al., 2011). Pre-anesthetic treatment with midazolam was found to be associated with minimal adverse cardiovascular effects (Dzikiti et al., 2011). Propofol (2, 6-di-isopropylphenol) is a phenolic compound commonly used in veterinary and human anesthetic practice that has capacity to induce smooth anesthesia and permit fast and uneventful recovery (Hall et al., 2001; Bodh et al., 2013). Propofol alone or in combination with other anesthetic agents had been proved a good anesthetic agent for induction and maintenance of anesthesia (Mattos et al., 2013; Chui et al., 2014; Dahi et al., 2015). Ketamine is 2(O-chlorophenyl)-2-(methylamine)-cyclohexane chloride that produced a dissociative state of anesthesia (Mama et al., 2005). Ketamine produced analgesic, anesthetic and cataleptic effects without any hypnosis (Clarke et al., 
2014). The muscle relaxant limiting property of ketamine can be improved by mixing it with benzodiazepines (Barletta et al., 2011).

To the knowledge of the author, currently, very little scientific findings have been reported regarding dose sparing effects of different sedatives on recently available induction agents as well as the impact of these agents on the quality of general anesthesia in goats. Hence, six combinations of these pre-anesthetic and anesthetic agents were made keeping in view their pharmacokinetics, pharmacodynamics and their safety margin in different species. The five different combinations included in present study had already been trialed in canines, equines and bovines but very few in caprines. In addition to these already trialed combinations in different species including goats, a new combination (detomidine+midazolam+propofol+ketamine) is first ever being trialed in goats. The objective of this study was to investigate the effects of detomidine and midazolam administered alone or combined, on induction dose and quality of propofol and ketamine anesthesia in goats. This research was initiated to answer if detomidine and midazolam administered alone or combined as a coinduction agents, could reduce the dose of propofol and ketamine in goats. Furthermore, if a reduction in dose was achieved, an additional aim was to determine if it would also impact on the quality of general anesthesia and ameliorate any potential negative cardio-pulmonary effects of these induction agents.

\section{MATERIALS AND METHODS}

The protocol for this study was approved by Ethical Review Committee, University of Veterinary \& Animal Sciences (UVAS), Lahore (No. DR/551). The experiment was conducted on twelve (12) healthy female goats aged between 08-12 months with average body weight of 24-30 kg. The experimental animals were procured form local market. They were kept at Institute of Continuing Education \& Extension (ICE \& E, UVAS Ravi Campus, Pattoki, Pakistan) for twenty one (21) days as adaptation period. During this period, they were served with feed and water. They were dewormed by giving Albendazole @10 mg/kg two weeks before experiment. Clinico-physiological parameters were also noted regularly throughout the period of acclimatization. A day before the experimental trial, the animals were shifted to Surgery Section, A-block Ravi campus Pattoki. All the animals were deprived of feed and water for 12 hours before sedation or anesthesia (Adetunji et al., 2002). The animals were weighed 30 minutes before the experiment using an electronic scale (Jadever ${ }^{\circledR}$ Richter Scale, Jadever, Scale Co. Ltd, Taipei, Taiwan). The left jugular vein site was prepared aseptically for administration of drugs. The control dose of propofol used for induction of anesthesia was chosen based on previous study (Prassinos et al., 2005). The goats were randomly divided into six groups (A, B, C, D, E and F) comprising two animals each. Sedative, analgesic, dose sparing, anesthetic and clinico-physiological parameters were evaluated before sedation, after sedation and during anesthesia to total recovery period.

\section{Pre-anesthetic \& Anesthetic Medication in Goats.}

\begin{tabular}{lll}
\hline Groups & Pre-anesthetic medication & Induction \\
\hline Group A & Normal Saline @ $10 \mathrm{ml} /$ whole body weight & Propofol @ $4.20 \pm 0.10 \mathrm{mg} / \mathrm{kg}$ body weight \\
Group B & Detomidine @ $15 \mu \mathrm{g} / \mathrm{kg}$ body weight & Different doses of Propofol \\
Group C & Detomidine @ $15 \mu \mathrm{g} / \mathrm{kg}$ body weight & Different doses of Ketamine \\
Group D & Midazolam @ $0.25 \mathrm{mg} / \mathrm{kg}$ body weight & Different doses of Propofol \\
Group E & Midazolam @ $0.25 \mathrm{mg} / \mathrm{kg}$ body weight & Different doses of Ketamine \\
Group F & Combination of detomidine+ midazolam & Different doses of combination of Propofol+ \\
& $@ 7 \mu \mathrm{g} / \mathrm{kg}+0.12 \mathrm{mg} / \mathrm{kg}$ body weight & Ketamine \\
\hline
\end{tabular}

\section{Parameter evaluated:}

Sedation: The degree of sedation was noted by observing behavioral changes fifteen minutes after pre-anesthetic treatment and was graded as following (Kalhoro et al., 2000):

$1=$ no sedation (animal alert with all reflexes present)

$2=$ mild sedation (animal dull with minimum reduction in reflexes)

$3=$ moderate sedation (animal calm with weak reflexes and partially closure of eyelids)
4 = deep sedation (animal recumbent with very weak reflexes and complete closure of eyelids)

Anesthetic parameters: Anesthesia parameters (induction quality, muscle relaxation quality, recovery quality, induction time, induction dose, \% age reduction in induction dose and duration of anesthesia) were recorded at 5 minutes interval post induction to throughout observation period. Quality of sedation, analgesia, induction, muscle relaxation and recovery were evaluated subjectively during anesthesia by using score card (Table 1). 
Clinico-physiological parameters: Clinicophysiological parameters (heart rate, respiratory rate and rectal temperature ${ }^{\circ} \mathrm{F}$ ) were recorded at $0 \mathrm{~min}$ before sedation, $15 \mathrm{~min}$ after sedation, $1 \mathrm{~min}$ after anesthetic induction and then every 5 minutes during anesthesia to total recovery period. The heart rate (beats/minute) was measured with Littman stethoscope (Littman Classic II, USA). The respiratory rate (breaths/min) was noted by observing the thoraco-abdominal movements. Rectal temperature $\left({ }^{\circ} \mathrm{F}\right)$ was recorded using Medicare digital thermometer (distributed by MANA \& Co, Pakistan).

Statistical Analysis: The collected data were arranged for dependent variables such as sedation \& analgesia quality, induction quality, muscle relaxation quality and recovery quality. Treatment groups were considered as independent variables. Anesthetic and clinicophysiological parameters are much dependent on postmedication time interval. To rule out such effects, data were also arranged for time effects in a single treatment group. Further, analysis was performed using one-way analysis of variance (ANOVA) technique in Statistical Analysis Software (SAS) 9.1 version at $(\mathrm{p} \leq 0.05)$. Significant differences among means were separated through Duncan's Multiple Range test.

\section{RESULTS}

Sedation and Analgesia: The statistical analysis showed significant differences $(\mathrm{p} \leq 0.05)$ among the treatment groups for sedation and analgesia, induction dose, $\%$ age reduction in induction, induction time, induction score, muscle relaxation score, recovery score, and duration of anesthesia (Table 2). The sedation and analgesia scores were found significantly $(p \leq 0.05)$ higher in group $F$ $(4.00 \pm 0.00)$ than control group $(1.00 \pm 0.00)$ and other treatment groups $(2.00 \pm 0.00)$. The sedation and analgesia scores in groups B, C, D and E were found $(2.00 \pm 0.00)$ for each that differed non-significantly $(p>0.05)$ from each other.

Anesthetic parameters: The mean value of induction dose was found significantly $(\mathrm{p} \leq 0.05)$ lower in group $\mathrm{F}$ $(2.70 \pm 0.00 \mathrm{mg} / \mathrm{kg})$ than control group A $(4.20 \pm 0.10$ $\mathrm{mg} / \mathrm{kg}$ ) and other treatment groups. While, the mean value of induction dose required in group $\mathrm{B}$ and $\mathrm{C}$ were found $(3.25 \pm 0.05 \mathrm{mg} / \mathrm{kg})$ and $(3.40 \pm 0.00 \mathrm{mg} / \mathrm{kg})$ respectively that differed non-significantly $(\mathrm{p}>0.05)$ from each other. Similarly, the mean value of induction dose required in group D and $\mathrm{E}$ were found $(3.50 \pm 0.00 \mathrm{mg} / \mathrm{kg})$ and $(3.60 \pm 0.00 \mathrm{mg} / \mathrm{kg})$ respectively that also differed non-significantly $(\mathrm{p}>0.05)$ from each other. The maximum \% age reduction in induction dose was noted in group $F(35.68 \pm 1.53 \%)$ that differed significantly $(\mathrm{p} \leq 0.05)$ than control group and other treatment groups. The \% age reduction in induction dose in group $\mathrm{B}$ and $\mathrm{C}$ were found $(22.60 \pm 0.65 \%)$ and $(19.00 \pm 1.93 \%)$ respectively that differed non-significantly $(\mathrm{p}>0.05)$ from each other. Similarly, the \% age reduction in induction dose in group D and E were found $(16.62 \pm 1.99 \%)$ and $(14.24 \pm 2.04 \%)$ respectively that also differed nonsignificantly $(p>0.05)$ from each other. The induction

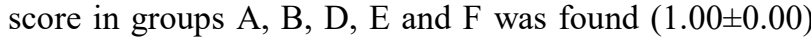
indicating smooth induction that differed significantly $(\mathrm{p} \leq 0.05)$ from group $\mathrm{C}(3.00 \pm 0.00)$ indicating rough induction. The muscle relaxation score was found $(1.00 \pm 0.00)$ in group F indicating excellent, $(2.00 \pm 0.00)$ in groups $\mathrm{A}, \mathrm{B}, \mathrm{D}$ and $\mathrm{E}$ indicating good and $(3.00 \pm 0.00)$ in group $\mathrm{C}$, indicating poor muscle relaxation quality. All treatment groups differed significantly $(\mathrm{p} \leq 0.05)$ from each other. The recovery score in groups $\mathrm{A}, \mathrm{B}, \mathrm{E}$ and $\mathrm{F}$ was found $(1.00 \pm 0.00)$ indicating smooth, $(2.00 \pm 0.00)$ in group D, indicating fair and $(3.00 \pm 0.00)$ in group $\mathrm{C}$, indicating poor recovery quality. All treatment groups differed significantly $(\mathrm{p} \leq 0.05)$ from each other. The maximum duration of anesthesia was produced in group F $(9.22 \pm 0.09 \mathrm{~min})$ that differed significantly $(\mathrm{p} \leq 0.05)$ than control group and other treatment groups. The average duration of anesthesia in group $\mathrm{B}$ and $\mathrm{C}$ was found $(5.76 \pm 0.64 \mathrm{~min})$ and $(6.42 \pm 0.90 \mathrm{~min})$ respectively that differed non-significantly $(\mathrm{p}>0.05)$ from each other. Similarly, the average duration of anesthesia in group $D$ and $E$ was found $(6.89 \pm 0.33 \mathrm{~min})$ and $(5.77 \pm 0.44 \mathrm{~min})$ respectively that also differed non-significantly $(p>0.05)$ from each other.

Clinico-physiological parameters: Of the clinicophysiological parameters assessed, the statistical analysis showed significant $(\mathrm{p} \leq 0.05)$ differences for heart rate, respiratory rate and non-significant $(p>0.05)$ differences for rectal temperature among or within all groups (Table $3,4,5)$. These parameters returned to normal values after recovery. The heart rate increased significantly $(\mathrm{p} \leq 0.05)$ within groups $\mathrm{A}, \mathrm{D}$ and $\mathrm{E}$ from base value to onwards till recovery with peak increase at 15, 30 and $25 \mathrm{~min}$ respectively. While, the heart rate decreased significantly $(\mathrm{p} \leq 0.05)$ within groups $\mathrm{B}, \mathrm{C}$ and $\mathrm{F}$ from base value to onwards till recovery with a peak decrease at 25,40 and $25 \mathrm{~min}$ respectively. The comparison among the treatment groups revealed that the heart rate differed nonsignificantly $(p>0.05)$ at 0 minute before sedation then differed significantly $(\mathrm{p} \leq 0.05)$ at 15 minute after sedation and 1 minute after induction to onwards till recovery in all groups (Table 3). Similarly, the respiratory rate decreased significantly $(\mathrm{p} \leq 0.05)$ within groups $\mathrm{A}, \mathrm{B}, \mathrm{C}, \mathrm{D}$ and $\mathrm{F}$ from base value to onwards till recovery with peak decrease at $25,25,40,40$ and $15 \mathrm{~min}$ respectively. Whereas, the respiratory rate increased significantly $(\mathrm{p} \leq 0.05)$ within group $\mathrm{E}$ with peak increase at $25 \mathrm{~min}$. While the comparison among the treatment groups revealed that respiratory rate differed non-significantly $(p>0.05)$ at 0 minute before sedation then differed significantly $(\mathrm{p} \leq 0.05)$ at 15 minute after sedation and 1 
minute after induction to onwards till recovery in all groups (Table 4). The rectal temperature also decreased non-significantly $(\mathrm{p}>0.05)$ within groups $\mathrm{A}, \mathrm{B}, \mathrm{C}, \mathrm{D}, \mathrm{E}$ and $\mathrm{F}$ from base value to onwards till recovery with a peak decrease at 20,5,5,30,15 and 10 min respectively. The comparison among the treatment groups revealed that the rectal temperature differed non-significantly $(p>0.05)$ at 0 minute before sedation, 15 minute after sedation and 1 minute after induction to onwards till recovery in all groups (Table 5).

Adverse effects: Mild and hyper-salivation was observed in group A and B respectively. While, mild salivation along-with snoring, regurgitation, ataxia and incoordination was observed in group $\mathrm{C}$ and $\mathrm{D}$. Urination and mild ataxia was observed in group E. However, no adverse effects were noticed in group F.

Table 1. A score card used for assessment of quality of different anesthetic parameters.

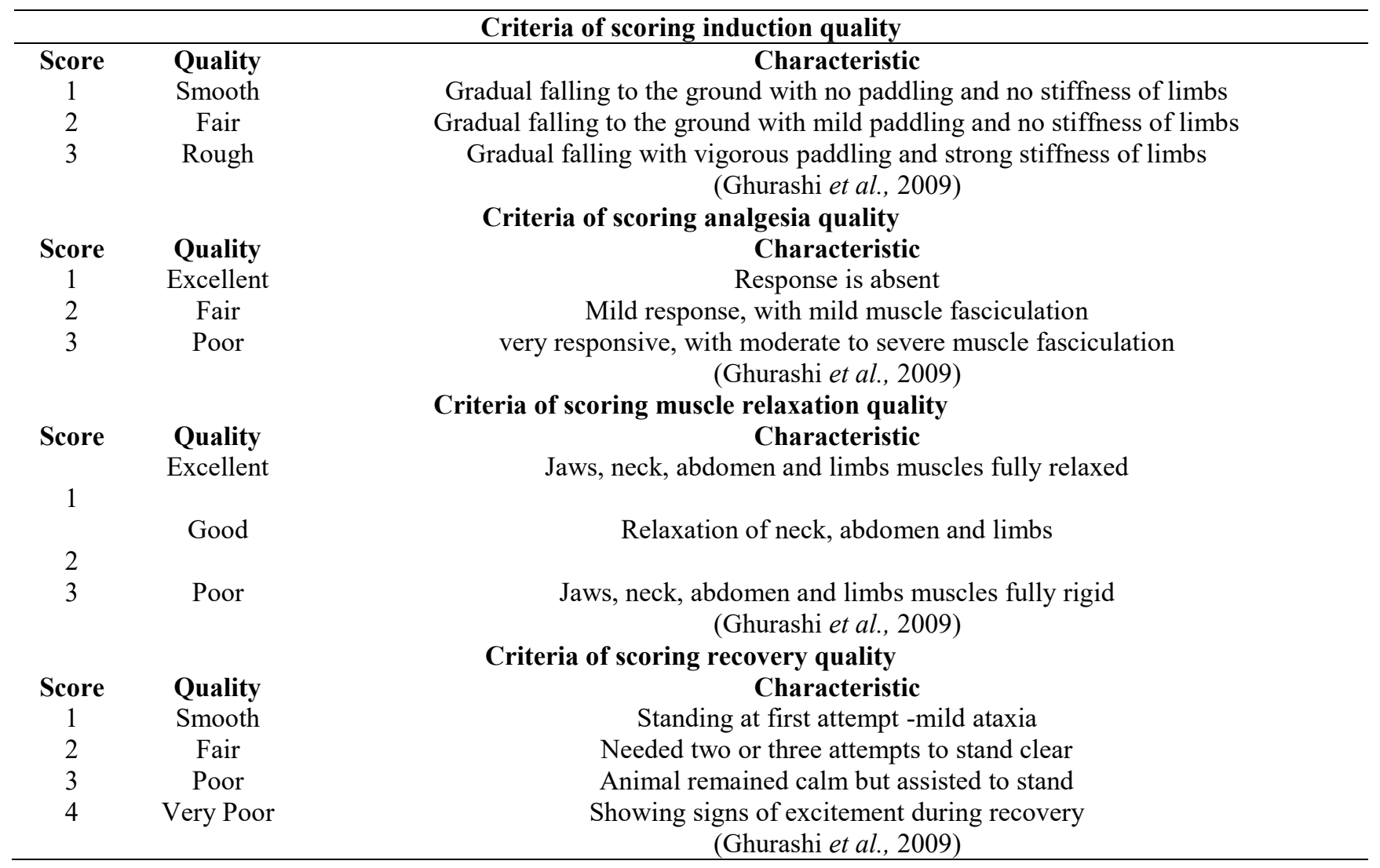


Table 2. Sedative, analgesic, dose sparing and anesthetic assessment of normal saline@10ml/whole b. wt., detomidine@15 $\mu$ g/kg, midazolam @ 0.25 $\mathrm{mg} / \mathrm{kg}$ and combination of detomidine and midazolam $@ 7 \mathrm{gg} / \mathrm{kg}+0.12 \mathrm{mg} / \mathrm{kg}$, on induction doses of propofol and $\mathrm{ketamine} \mathrm{in} \mathrm{goat.}$

\begin{tabular}{|c|c|c|c|c|c|c|c|c|c|c|}
\hline & Treatment groups & Sed. score & $\begin{array}{l}\text { Anal. } \\
\text { Score }\end{array}$ & I.D (mg/kg) & $\begin{array}{l}\text { (\%) Red in } \\
\text { I.D }\end{array}$ & I.Q & M.R.Q & $\begin{array}{c}\text { I.T } \\
\text { (Sec) }\end{array}$ & $\begin{array}{c}\text { D. o. A } \\
\text { (min) }\end{array}$ & Rec. Q \\
\hline $\mathbf{A}$ & N.S + Prop & $1.00 \pm 0.00^{\mathrm{c}}$ & $1.00 \pm 0.00^{\mathrm{c}}$ & $4.20 \pm 0.10^{\mathrm{a}}$ & $0.00 \pm 0.00^{\mathrm{d}}$ & $1.00 \pm 0.00^{\mathrm{b}}$ & $2.00 \pm 0.00^{\mathrm{b}}$ & $24.00 \pm 1.00^{\mathrm{d}}$ & $2.31 \pm 0.24^{\mathrm{c}}$ & $1.00 \pm 0.00^{\mathrm{c}}$ \\
\hline B & Det + Prop & $2.00 \pm 0.00^{\mathrm{b}}$ & $2.00 \pm 0.00^{\mathrm{b}}$ & $3.25 \pm 0.05^{\mathrm{d}}$ & $22.60 \pm 0.65^{b}$ & $1.00 \pm 0.00^{\mathrm{b}}$ & $2.00 \pm 0.00^{\mathrm{b}}$ & $42.00 \pm 4.00^{c}$ & $5.76 \pm 0.64^{b}$ & $1.00 \pm 0.00^{\mathrm{c}}$ \\
\hline $\mathbf{C}$ & Det + Ket & $2.00 \pm 0.00^{\mathrm{b}}$ & $2.00 \pm 0.00^{\mathrm{b}}$ & $3.40 \pm 0.00^{\mathrm{cd}}$ & $19.00 \pm 1.93^{b c}$ & $3.00 \pm 0.00^{\mathrm{a}}$ & $3.00 \pm 0.00^{\mathrm{a}}$ & $55.00 \pm 4.00^{\mathrm{b}}$ & $6.42 \pm 0.90^{\mathrm{b}}$ & $3.00 \pm 0.00^{\mathrm{a}}$ \\
\hline D & Mid + Prop & $2.00 \pm 0.00^{\mathrm{b}}$ & $2.00 \pm 0.00^{\mathrm{b}}$ & $3.50 \pm 0.00^{\mathrm{bc}}$ & $16.62 \pm 1.99^{c}$ & $1.00 \pm 0.00^{\mathrm{b}}$ & $2.00 \pm 0.00^{\mathrm{b}}$ & $52.00 \pm 2.00^{\mathrm{b}}$ & $6.89 \pm 0.33^{\mathrm{b}}$ & $2.00 \pm 0.00^{\mathrm{b}}$ \\
\hline $\mathbf{E}$ & Mid + Ket & $2.00 \pm 0.00^{\mathrm{b}}$ & $2.00 \pm 0.00^{\mathrm{b}}$ & $3.60 \pm 0.00^{\mathrm{b}}$ & $14.24 \pm 2.04^{\mathrm{c}}$ & $1.00 \pm 0.00^{\mathrm{b}}$ & $2.00 \pm 0.00^{\mathrm{b}}$ & $74.00 \pm 2.00^{\mathrm{a}}$ & $5.77 \pm 0.44^{\mathrm{b}}$ & $1.00 \pm 0.00^{\mathrm{c}}$ \\
\hline $\mathbf{F}$ & $\begin{array}{c}\text { Det }+ \text { Mid }+ \text { Prop }+ \text { Ket } \\
\text { p-value }\end{array}$ & $\begin{array}{c}4.00 \pm 0.00^{\mathrm{a}} \\
<.0001\end{array}$ & $\begin{array}{l}4.00 \pm 0.00^{\mathrm{a}} \\
<.0001\end{array}$ & $\begin{array}{l}2.70 \pm 0.00^{\mathrm{e}} \\
<.0001\end{array}$ & $\begin{array}{l}35.68 \pm 1.53^{\mathrm{a}} \\
<.0001\end{array}$ & $\begin{array}{c}1.00 \pm 0.00^{\mathrm{b}} \\
0.0001\end{array}$ & $\begin{array}{l}1.00 \pm 0.00^{\mathrm{c}} \\
<.0001\end{array}$ & $\begin{array}{l}19.00 \pm 1.00^{\mathrm{d}} \\
<.0001\end{array}$ & $\begin{array}{c}9.22 \pm 0.09^{\mathrm{a}} \\
0.0013\end{array}$ & $\begin{array}{l}1.00 \pm 0.00^{\mathrm{c}} \\
<.0001\end{array}$ \\
\hline
\end{tabular}

Sed. is sedation score; Anal. is analgesia ; I.D is induction dose in ( $\mathrm{mg} / \mathrm{kg}) ; \%$ Red in I.D is \% age reduction in induction dose; I.Q is induction quality; M.R.Q is muscle relaxation quality; I.T is induction time; D.o.A is duration of anesthesia; Rec. Q is recovery quality.

Table 3. Mean value $( \pm$ S.E.) of heart rate (beats/minute) following premedication with normal saline $@ 10 \mathrm{ml} / \mathrm{whole}$ b. wt., detomidine $@ 15 \mu \mathrm{m} / \mathrm{kg}$, midazolam@ $0.25 \mathrm{mg} / \mathrm{kg}$ and combination of detomidine and midazolam $@ 7 \mu \mathrm{g} / \mathrm{kg}+0.12 \mathrm{mg} / \mathrm{kg}$ and induction with different doses of propofol and ketamine in goat.

\begin{tabular}{|c|c|c|c|c|c|c|c|c|c|c|c|c|c|}
\hline & \multirow{2}{*}{$\begin{array}{l}\text { Treatment } \\
\text { groups }\end{array}$} & \multicolumn{2}{|c|}{ Post-sedation } & \multicolumn{9}{|c|}{ Post-induction } & \multirow{2}{*}{$\begin{array}{c}\text { p- } \\
\text { valu } \\
\text { e }\end{array}$} \\
\hline & & 0 min & $15 \mathrm{~min}$ & $1 \mathrm{~min}$ & $5 \mathrm{~min}$ & $10 \mathrm{~min}$ & $15 \mathrm{~min}$ & 20 min & $25 \mathrm{~min}$ & $30 \mathrm{~min}$ & $35 \mathrm{~min}$ & $40 \mathrm{~min}$ & \\
\hline $\mathbf{A}$ & $\begin{array}{l}\text { N.S + } \\
\text { Prop }\end{array}$ & $\begin{array}{c}59.00 \pm 1 . \\
00^{\mathrm{eA}}\end{array}$ & $\begin{array}{c}60.00 \pm 1 . \\
00^{\mathrm{eB}}\end{array}$ & $\begin{array}{c}83.00 \pm 3 . \\
00^{\mathrm{dA}}\end{array}$ & $\begin{array}{c}97.00 \pm 1 . \\
00^{\mathrm{cA}}\end{array}$ & $\begin{array}{c}101.50 \pm 0 \\
.50^{\mathrm{bcA}}\end{array}$ & $\begin{array}{c}107.00 \pm 1 \\
00^{\mathrm{aA}}\end{array}$ & $\begin{array}{c}103.00 \pm 1 \\
.00^{\mathrm{abA}}\end{array}$ & $\begin{array}{c}106.00 \pm 1 \\
.00^{\mathrm{abA}}\end{array}$ & & & & $\begin{array}{c}<.00 \\
01\end{array}$ \\
\hline $\mathbf{B}$ & $\begin{array}{l}\text { Det }+ \\
\text { Prop }\end{array}$ & $\begin{array}{c}63.00 \pm 1 . \\
00^{\mathrm{aAB}}\end{array}$ & $\begin{array}{c}55.00 \pm 2 . \\
00^{\mathrm{bcC}}\end{array}$ & $\begin{array}{c}56.50 \pm 0 . \\
50^{\mathrm{bcC}}\end{array}$ & $\begin{array}{c}57.50 \pm 0 . \\
50^{\mathrm{bC}}\end{array}$ & $\begin{array}{c}53.50 \pm 1 \\
50^{\mathrm{bcc}}\end{array}$ & $\begin{array}{c}55.00 \pm 1.0 \\
0^{\mathrm{bcD}}\end{array}$ & $\begin{array}{c}53.50 \pm 0 . \\
50^{\mathrm{bcD}}\end{array}$ & $\begin{array}{c}52.50 \pm 1 \\
50^{\mathrm{cD}}\end{array}$ & & & & $\begin{array}{c}0.00 \\
46\end{array}$ \\
\hline $\mathbf{C}$ & Ket & $\begin{array}{c}61.00 \pm 1 \\
00^{\mathrm{aAB}}\end{array}$ & $\begin{array}{c}46.00 \pm 1 \\
00^{\mathrm{eD}}\end{array}$ & $\begin{array}{c}54.00 \pm 1 \\
00^{\mathrm{bC}}\end{array}$ & $\begin{array}{c}51.00 \pm 1 \\
00^{\text {bcdD }}\end{array}$ & $\begin{array}{c}52.00 \pm 1 \\
00^{\mathrm{bcC}}\end{array}$ & $\begin{array}{c}50.50 \pm 2.5 \\
0^{\text {bcdDE }}\end{array}$ & $\begin{array}{c}51.00 \pm 0 . \\
00^{\text {bcdD }}\end{array}$ & $\begin{array}{c}52.00 \pm 0 . \\
00^{\mathrm{bcD}}\end{array}$ & $\begin{array}{c}49.00 \pm 0 . \\
00^{\text {cdeB }}\end{array}$ & $\begin{array}{c}48.50 \pm 0 . \\
50^{\text {cdeB }}\end{array}$ & $\begin{array}{c}48.00 \pm 0 . \\
00^{\mathrm{deB}}\end{array}$ & $\begin{array}{c}<.00 \\
01\end{array}$ \\
\hline D & $\begin{array}{l}\text { Mid + } \\
\text { Prop }\end{array}$ & $\begin{array}{c}62.00 \pm 2 . \\
00^{\mathrm{fAB}}\end{array}$ & $\begin{array}{c}67.00 \pm 1 . \\
00^{\mathrm{eA}}\end{array}$ & $\begin{array}{c}70.00 \pm 1 . \\
00^{\mathrm{eB}}\end{array}$ & $\begin{array}{c}77.00 \pm 1 . \\
00^{\mathrm{dB}}\end{array}$ & $\begin{array}{c}80.00 \pm 1 \\
00^{\mathrm{cdB}}\end{array}$ & $\begin{array}{c}83.00 \pm 1.0 \\
0^{\mathrm{cB}}\end{array}$ & $\begin{array}{c}87.00 \pm 1 . \\
00^{\mathrm{bB}}\end{array}$ & $\begin{array}{c}87.00 \pm 1 \\
00^{\mathrm{bB}}\end{array}$ & $\begin{array}{c}93.00 \pm 1 . \\
00^{\mathrm{aA}}\end{array}$ & $\begin{array}{c}92.00 \pm 0 . \\
00^{\mathrm{aA}}\end{array}$ & $\begin{array}{c}92.00 \pm 0 . \\
00^{\mathrm{aA}}\end{array}$ & $\begin{array}{c}<.00 \\
01\end{array}$ \\
\hline $\mathbf{E}$ & Ket ${ }^{\text {Mid + }}$ & $\begin{array}{c}59.00 \pm 1 \\
00^{\mathrm{eB}}\end{array}$ & $\begin{array}{c}66.00 \pm 2 . \\
00^{\mathrm{dA}}\end{array}$ & $\begin{array}{c}71.00 \pm 1 \\
00^{\mathrm{cB}}\end{array}$ & $\begin{array}{c}74.00 \pm 1 \\
00^{\mathrm{bcB}}\end{array}$ & $\begin{array}{c}76.00 \pm 2 . \\
00^{\mathrm{abB}}\end{array}$ & $\begin{array}{c}76.00 \\
\pm 1.00^{\mathrm{aC}}\end{array}$ & $\begin{array}{c}79.00 \pm 1 \\
00^{\mathrm{aC}}\end{array}$ & $\begin{array}{c}80.00 \pm 1 \\
00^{\mathrm{aC}}\end{array}$ & & & & $\begin{array}{c}<.00 \\
01\end{array}$ \\
\hline $\mathbf{F}$ & $\begin{array}{c}\text { Det+Mid+Pr } \\
\text { op+Ket } \\
\text { p-value }\end{array}$ & $\begin{array}{c}64.00 \pm 0 . \\
00^{\mathrm{aA}} \\
0.0946\end{array}$ & $\begin{array}{c}54.50 \pm 0 . \\
50^{\mathrm{cC}} \\
0.0003\end{array}$ & $\begin{array}{c}58.00 \pm 0 . \\
00^{\mathrm{bC}} \\
<.0001\end{array}$ & $\begin{array}{c}54.00 \pm 1 \\
00^{\mathrm{cD}} \\
<.0001\end{array}$ & $\begin{array}{c}53.00 \pm 1 \\
00^{\mathrm{cC}} \\
<.0001\end{array}$ & $\begin{array}{c}48.00 \pm 0.0 \\
0^{\mathrm{dE}} \\
<.0001\end{array}$ & $\begin{array}{c}46.00 \\
\pm 0.00^{\mathrm{dF}} \\
<.0001\end{array}$ & $\begin{array}{c}46.00 \pm 1 \\
00^{\mathrm{dE}} \\
<.0001\end{array}$ & 0.0005 & 0.0001 & $<.0001$ & $\begin{array}{c}<.00 \\
01\end{array}$ \\
\hline
\end{tabular}

Different superscripts within a column show significant differences among treatment groups

Different superscripts within a row show significant differences at different time intervals 


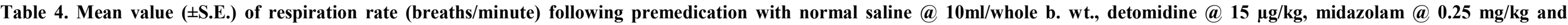
combination of detomidine and midazolam $(a) 7 \mathrm{~g} / \mathrm{kg}+0.12 \mathrm{mg} / \mathrm{kg}$ and induction with different doses of propofol and ketamine in $\mathrm{goat}$.

\begin{tabular}{|c|c|c|c|c|c|c|c|c|c|c|c|c|c|}
\hline \multirow{2}{*}{\multicolumn{2}{|c|}{ Treatment groups }} & \multicolumn{2}{|c|}{ Post-sedation } & \multicolumn{9}{|c|}{ Post-induction } & \multirow[t]{2}{*}{ p-value } \\
\hline & & $\mathbf{0} \min$ & $15 \min$ & 1 min & 5 min & $10 \mathrm{~min}$ & $15 \mathrm{~min}$ & $20 \mathrm{~min}$ & $25 \min$ & $30 \mathrm{~min}$ & $35 \min$ & $40 \mathrm{~min}$ & \\
\hline $\mathbf{A}$ & N.S + Prop & $17.00 \pm 1.00^{\mathrm{aA}}$ & $16.00 \pm 0.00^{\mathrm{aAB}}$ & $13.50 \pm 0.50^{\mathrm{bB}}$ & $13.50 \pm 0.50^{\mathrm{bB}}$ & $12.50 \pm 0.50^{\mathrm{bB}}$ & $12.50 \pm 0.50^{\mathrm{bB}}$ & $12.00 \pm 0.00^{\mathrm{bB}}$ & $12.00 \pm 0.00^{\mathrm{bB}}$ & & & & 0.0006 \\
\hline B & Det + Prop & $17.00 \pm 1.00^{\mathrm{aA}}$ & $14.00 \pm 0.00^{\mathrm{bC}}$ & $13.00 \pm 0.00 \mathrm{~b}^{\mathrm{cB}}$ & $12.50 \pm 0.50^{\mathrm{cdBC}}$ & $12.00 \pm 0.00^{\mathrm{cdB}}$ & $12.00 \pm 0.00^{\mathrm{cdB}}$ & $11.50 \pm 0.50^{\mathrm{cdB}}$ & $11.00 \pm 0.00^{\mathrm{dB}}$ & & & & 0.0002 \\
\hline C & Det + Ket & $16.50 \pm 0.50^{\mathrm{aA}}$ & $13.50 \pm 0.50^{\mathrm{bC}}$ & $12.50 \pm 0.50 \mathrm{~b}^{\mathrm{cBC}}$ & $12.50 \pm 0.50^{\mathrm{bcBC}}$ & $12.00 \pm 0.00^{\mathrm{cdB}}$ & $11.50 \pm 0.50^{\mathrm{cdB}}$ & $11.50 \pm 0.50^{\mathrm{cdB}}$ & $11.50 \pm 0.50^{\mathrm{cdB}}$ & $11.00 \pm 0.00^{\mathrm{d}}$ & $11.00 \pm 0.00^{\mathrm{d}}$ & $11.00 \pm 0.00^{\mathrm{d}}$ & $<.0001$ \\
\hline D & Mid +Prop & $17.00 \pm 1.00^{\mathrm{aA}}$ & $14.50 \pm 0.50^{\mathrm{bBC}}$ & $13.50 \pm 0.50^{\mathrm{bcB}}$ & $12.50 \pm 0.50^{\mathrm{cdBC}}$ & $12.00 \pm 0.00^{\mathrm{cdB}}$ & $11.50 \pm 0.50^{\mathrm{dB}}$ & $11.50 \pm 0.50^{\mathrm{dB}}$ & $11.00 \pm 0.00^{\mathrm{dB}}$ & $11.50 \pm 0.50^{\mathrm{d}}$ & $11.00 \pm 0.00^{\mathrm{d}}$ & $11.00 \pm 0.00^{\mathrm{d}}$ & $<.0001$ \\
\hline $\mathbf{E}$ & Mid +Ket & $15.00 \pm 1.00^{\mathrm{dA}}$ & $17.00 \pm 1.00^{\mathrm{cA}}$ & $18.00 \pm 0.00 \mathrm{~b}^{\mathrm{cA}}$ & $19.50 \pm 0.50^{\mathrm{abA}}$ & $19.50 \pm 0.50^{\mathrm{abA}}$ & $20.00 \pm 0.00^{\mathrm{abA}}$ & $20.50 \pm 0.50^{\mathrm{aA}}$ & $20.50 \pm 0.50^{\mathrm{aA}}$ & & & & 0.0020 \\
\hline F & $\begin{array}{c}\text { Det+Mid+Prop+Ket } \\
\text { p-value }\end{array}$ & $\begin{array}{c}14.00 \pm 0.00^{\mathrm{aA}} \\
0.1677\end{array}$ & $\begin{array}{c}11.00 \pm 0.00^{\mathrm{cD}} \\
0.0016\end{array}$ & $\begin{array}{c}11.50 \pm 0.50 \mathrm{~b}^{\mathrm{cC}} \\
0.0003\end{array}$ & $\begin{array}{c}11.50 \pm 0.50^{\mathrm{bcc}} \\
0.0002\end{array}$ & $\begin{array}{l}12.00 \pm 0.00^{\mathrm{bcB}} \\
<.0001\end{array}$ & $\begin{array}{l}12.50 \pm 0.50^{\mathrm{bB}} \\
<.0001\end{array}$ & $\begin{array}{l}11.50 \pm 0.50^{\mathrm{bcB}} \\
<.0001\end{array}$ & $\begin{array}{l}12.00 \pm 0.00^{\mathrm{bcB}} \\
<.0001\end{array}$ & & & & 0.0072 \\
\hline
\end{tabular}

Different superscripts within a column show significant differences among treatment groups

Different superscripts within a row show significant differences at different time intervals

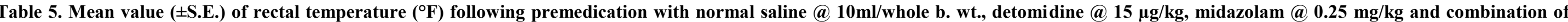
detomidine and midazolam @ $7 \mu \mathrm{g} / \mathrm{kg}+0.12 \mathrm{mg} / \mathrm{kg}$ and induction with different doses of propofol and ketamine in goat.

\begin{tabular}{|c|c|c|c|c|c|c|c|c|c|c|c|c|c|}
\hline & \multirow[t]{2}{*}{ Treatment groups } & \multicolumn{2}{|c|}{ Post-sedation } & \multicolumn{9}{|c|}{ Post-induction } & \multirow[t]{2}{*}{ p-value } \\
\hline & & 0 min & $15 \mathrm{~min}$ & $1 \mathrm{~min}$ & $5 \mathrm{~min}$ & $10 \mathrm{~min}$ & $15 \mathrm{~min}$ & $20 \mathrm{~min}$ & $25 \mathrm{~min}$ & $30 \mathrm{~min}$ & $35 \mathrm{~min}$ & $40 \mathrm{~min}$ & \\
\hline $\mathbf{A}$ & N.S + Prop & $102.50 \pm 0.05$ & $102.05 \pm 0.05$ & $101.90 \pm 0.00$ & $101.85 \pm 0.05$ & $101.80 \pm 0.10$ & $101.80 \pm 0.10$ & $101.80 \pm 0.00$ & $101.85 \pm 0.05$ & & & & 0.0733 \\
\hline B & Det + Prop & $102.10 \pm 0.00$ & $101.95 \pm 0.05$ & $101.85 \pm 0.05$ & $101.75 \pm 0.05$ & $101.75 \pm 0.05$ & $101.80 \pm 0.10$ & $101.85 \pm 0.05$ & $101.80 \pm 0.10$ & & & & 0.0562 \\
\hline $\mathbf{C}$ & Det + Ket & $102.50 \pm 0.05$ & $101.85 \pm 0.05$ & $101.80 \pm 0.00$ & $101.60 \pm 0.10$ & $101.70 \pm 0.10$ & $101.75 \pm 0.05$ & $101.75 \pm 0.15$ & $101.65 \pm 0.05$ & $101.75 \pm 0.05$ & $101.80 \pm 0.10$ & $101.85 \pm 0.05$ & 0.0928 \\
\hline D & Mid + Prop & $102.15 \pm 0.05$ & $101.80 \pm 0.00$ & $101.75 \pm 0.05$ & $101.65 \pm 0.05$ & $101.65 \pm 0.05$ & $101.70 \pm 0.10$ & $101.70 \pm 0.10$ & $101.70 \pm 0.20$ & $101.60 \pm 0.10$ & $101.70 \pm 0.10$ & $101.80 \pm 0.10$ & 0.0845 \\
\hline $\mathbf{E}$ & Mid + Ket & $102.10 \pm 0.00$ & $101.95 \pm 0.05$ & $101.85 \pm 0.05$ & $101.75 \pm 0.05$ & $101.80 \pm 0.10$ & $101.75 \pm 0.05$ & $101.85 \pm 0.05$ & $101.80 \pm 0.10$ & & & & 0.0572 \\
\hline $\mathbf{F}$ & $\begin{array}{c}\text { Det+Mid+Prop+Ket } \\
\text { p-value }\end{array}$ & $\begin{array}{c}102.10 \pm 0.00 \\
0.4339\end{array}$ & $\begin{array}{c}101.85 \pm 0.05 \\
0.0595\end{array}$ & $\begin{array}{c}101.75 \pm 0.05 \\
0.1825\end{array}$ & $\begin{array}{c}101.75 \pm 0.05 \\
0.2013\end{array}$ & $\begin{array}{c}101.75 \pm 0.05 \\
0.7379\end{array}$ & $\begin{array}{c}101.75 \pm 0.15 \\
0.9733\end{array}$ & $\begin{array}{c}101.80 \pm 0.10 \\
0.8127\end{array}$ & $\begin{array}{c}101.80 \pm 0.00 \\
0.7531\end{array}$ & 0.3118 & 0.5528 & 0.6985 & 0.0953 \\
\hline
\end{tabular}

Different superscripts within a column show significant differences among treatment groups

Different superscripts within a row show significant differences at different time intervals 


\section{DISCUSSION}

In the present study, detomidine and midazolam when administered combined, caused deep sedation and strong analgesia in goats. A deep level of sedation and strong analgesia was obtained probably due to synergism between detomidine and midazolam. The synergistic interaction between dexmedetomidine and midazolam was also reported by (Bol et al., 2000). Midazolam as a single agent has a mild sedative effect but it shows additive or synergistic activity when administered with other sedatives (Cwiek et al., 2009). The combination of sedative drugs reinforced the sedative and analgesic actions of the single drug (Itamoto et al., 2000). The deep sedation and strong analgesia observed in the present study was also confirmed by earlier studies following the administration of medetomidine-midazolam (Kojima et al., 2002; Ahmad et al., 2011; Le-Chevallier et al., 2019). Detomidine produced sedative effects by activating $\alpha-2$ adrenergic receptor and analgesia due to stimulation of receptors within the brain and spinal cord (Sinclair, 2003). The premedication with detomidine-midazolam followed by induction with propofol-ketamine caused maximum reduction in induction dose and \% age reduction in induction dose. The $\%$ age reduction in induction dose might be due to direct effects of premedication agents on induction agents. The maximum $\%$ age reduction in induction dose was obtained probably due to a synergistic action of detomidine-midazolam with propofol-ketamine. However, the synergic mechanism of action of detomidine-midazolam with propofol-ketamine is unknown. The combination of sedatives and anesthetic agents with different mechanisms of action has the benefit of using low doses of individual agents (Ukwueze et al., 2014). Similar findings were also previously recorded by Kojima et al. (2002) who reported that sedation with combination of medetomidine-midazolam showed maximum dose sparing effects on induction dose of thiopental and propofol in dogs

The combined therapy with detomidinemidazolam-propofol-ketamine resulted in significant $(\mathrm{p} \leq 0.05)$ increase in duration of anesthesia. The increase in duration of anesthesia might be due to the combined sedative effects of detomidine- midazolam on propofolketamine. The maximum increase in duration of anesthesia along-with smooth induction, excellent muscle relaxation and smooth recovery may be probably attributable through a synergistic action of detomidinemidazolam with propofol-ketamine. The combinations of various pre-anesthetic had been used to prolong the duration of anesthesia along with shorter recovery time, thereby improving quality of anaesthesia (Potliya et al., 2015). Similar findings were also reported in calves (Kilic, 2008) and dogs (Sahoo et al., 2018). The smooth induction recorded in most of the groups may be attributed to the combined muscle relaxing effect of detomidine, midazolam and propofol.

The sedation with detomidine-midazolam followed by induction with propofol-ketamine caused significant $(\mathrm{p} \leq 0.05)$ reduction in heart rate and respiratory rate and non-significant $(\mathrm{p}>0.05)$ reduction in rectal temperature among or within all groups. The reduction in heart rate, respiratory rate and rectal temperature was obtained probably due to direct depressant action of drugs on central nervous system in general and in particular respiratory centre and hypothalamic thermoregulatory center. Decrease in the values of physiological parameters was attributed due to decreased metabolic rate, muscle relaxation and direct depressant action on central nervous system (Kandpal et $a l ., 2005)$. It could also have resulted from the synergistic effect of detomidine-midazolam with propofol-ketamine. The detomidine-midazolam-ketamine when administered combined caused significant reduction in heart rate in calves (Kilic, 2008). The propofol-ketamine-xylazine when administered combined caused significant reduction in heart rate and respiratory rate in goat (Okwudili et al., 2014). Bradycardia is a common cardiovascular effect of detomidine (Tunio et al., 2016), midazolam (Mehlisch, 2002), propofol (Njoku, 2015). However, tachycardia induced by ketamine was masked by detomidine which was also reported by (Hopster et al., 2014). Respiratory depression has been reported as a classical complication of xylazine (Clarke and Trim, 2014), midazolam (Biboulet et al., 2010), propofol (Wiese et al., 2010) and ketamine (Clarke and Trim, 2014). Hypothermia is a common classical complication of detomidine (Maravi et al., 2018), midazolam (Bodh et al., 2013) and propofol (Bodh et al., 2013).

Of the adverse effects observed; mild and hypersalivation was noticed in animals treated with normal saline-propofol and detomidine-propofol respectively. While, mild salivation along-with snoring, regurgitation, ataxia and incoordination were observed in animals treated with detomidine-ketamine and midazolampropofol. Urination and mild ataxia were observed in animals treated with midazolam-ketamine. However, no adverse effects were observed in the animals treated with combination of detomidine-midazolam-propofolketamine. Salivation after induction with either propofol or ketamine in the present study might be due to delayed effects of detomidine.

Conclusion: It is concluded that the combined therapy with detomidine-midazolam-propofol-ketamine was found to be safe and economical for short surgical procedures in goats. The significant changes in heart rate, respiratory rate and non-significant changes in rectal temperature were noticed in all groups.

Acknowledgments: We fully acknowledge the efforts and support provided by the administration of 
Department of Clinical Medicine \& Surgery, University of Veterinary \& Animal Sciences, Lahore.

Conflict of Interest: There is no conflict of interest shown by any author.

\section{REFERENCES}

Ahmad, R.A., P. Kinjavdekar, H.P. Aithal, A.M. Pawde and D. Kumar (2011). Effects of midazolam or midazolam-fentanyl on sedation and analgesia produced by intramuscular dexmedetomidine in dogs. Asian J. Anim. Sci. 5(5): 302-316.

Adetunji, A., R.A. Ajadi, C.O. Adewoye and B.O. Oyemakinde (2002). Total intravenous anesthesia with propofol: repeat bolus versus continuous propofol infusion technique in xylazine-premedicated dogs. Isr. J. Vet. Med. 57(4): 139-144.

Bednarski, R., K. Grimm, R. Harvey, V.M. Lukasik, W.S. Penn, B. Sargent and K. Spelts (2011). AAHA anesthesia guidelines for dogs and cats. J. Am. Anim. Hosp. Assoc. 47(6): 377-385.

Bol, C.J., J.P. Vogelaar, J.P. Tang and J.W. Mandema (2000). Quantification of pharmacodynamic interactions between dexmedetomidine and midazolam in the rat. J. Pharmacol. Exp. Ther. 294(1): 347-355.

Barletta, M., B.R. Austin, J.C. Ko, M.E. Payton, A.B. Weil and T. Inoue (2011). Evaluation of dexmedetomidine and ketamine in combination with opioids as injectable anesthesia for castration in dogs. J. Am. Vet. Med. A. 238(9): 1159-1167.

Brighton, D.T. (2013). Intravenous anaesthesia in goats: a review. J. S. Afr. Vet. Assoc. 84(1): 1-8.

Biboulet, M., M. Lapeyre-Mestre and V. Gardette (2010). Comparison of benzodiazepine consumption according to type of buprenorphine use in a group of new users. Prat Organ Soins. 41(3): 205-213.

Bodh, D., K. Singh, J. Mohindroo, S.K. Mahajan, A. Anand and N.S. Saini (2013). Propofol and thiopentone sodium as induction agents in water buffaloes (Bubalus bubalis): a comparative study. J. Appl. Anim. Res. 41(3): 370-373.

Chui, J., R. Mariappan, J. Mehta, P. Manninen and L. Venkatraghavan (2014). Comparison of propofol and volatile agents for maintenance of anesthesia during elective craniotomy procedures: systematic review and metaanalysis. Can. J. Anaesth. 61(4): 347-356.

Clarke, K.W., C.M. Trim and L.W. Hall (2014). Veterinary Anaesthesia. 11th Ed. W.B. Saunders, Oxford. 80-148 p.
Clarke, K.W. and T.M. Trim (2014). Veterinary Anaesthesia. 11th Ed. Hacourt Publishers Ltd; (England). 146-150 p.

Cwiek, A., I. Balicki, D. Rozansk, I. Polkowska and M. Orzelski (2009). Propofol-induced inhalation anaesthesia in dogs after xylasine or xylasine and midazolam premedication. Med. Weter. 65(1): 29-32.

Dahi, M., F. Pourdanesh, S. Samieirad, G. Morad and A. Khojasteh (2015). Blood biomarkers alterations with administration of propofol for anaesthesia maintenance during long term oral and maxillofacial surgeries. Shahid Beheshti University Dental J. 33(1): 19-27.

Dzikiti, T.B., G.F. Stegmann, L.N. Dzikiti and L.J. Hellebrekers (2011). Effects of midazolam on isoflurane minimum alveolar concentration in goats. Small Rumin. Res. 97(1-3): 104-109.

El-Kammar, M.H., S.B. Gad and A.S. Korittum (2014). Evaluation of the Sedative, Analgesic, Physiological and Haematological Effects of Intravenous Detomidine, DetomidineButorphanol, Romifidine and RomifidineButorphanol in Baladi Goats. Glob. Vet. 12(1): 36-44.

Galatos, A.D. (2011). Anesthesia and analgesia in sheep and goats. Vet. Clin. N. Am. Food. A. 27(1): 4759.

Ghurashi, M.A.H., H.I. Seri, A.H. Bakheit, E.A.M. Ashwag and J.A. Abakar (2009). Evaluation of ketamine/diazepam anaesthesia for performing surgery in desert goats under field conditions. Aust. J. Basic. Appl. Sci. 3(2): 455-459.

Hall, L.W., K.W. Clarke and C.M. Trim (2001). Anaesthesia of sheep, goats and other herbivores. Vet. Anaesth. 10: 341-365.

Hendrickx, J. F., E.I. Eger, J.M. Sonner and S.L. Shafer (2008). Is synergy the rule? A review of anesthetic interactions producing hypnosis and immobility. Anesth. Analg. 107(2): 494-506.

Hopster, K., C. Müller, C. Hopster-Iversen, J. Stahl, K. Rohn and S. Kästner (2014). Effects of dexmedetomidine and xylazine on cardiovascular function during total intravenous anaesthesia with midazolam and ketamine and recovery quality and duration in horses. Vet. Anaesth. Analg. 41(1): 25-35.

Itamoto, K., Y. Hikasa, I. Sakonjyu, H. Itoh, T. Kakuta and K. Takase (2000). Anaesthetic and cardiopulmonary effects of balanced anaesthesia with medetomidine-midazolam and butorphanol in dogs. J Vet. Med. A. 47(7): 411-420.

Kalhoro, A.B., S.K. Shahani, A.B. Kachiwal, I.H. Kathio, A.Q. Memon and S.A. Soomro (2000). Physiological effects of medetomidine in buffalo 
calves. In Proc 3rd Asia Buffalo Conference, Kandy (Sri Lanka). 283-289 p.

Kilic, N. (2008). Cardiopulmonary, biochemical and haematological changes after detomidinemidazolam-ketamine anaesthesia in calves. B. Vet. I. Pulawy. 52(3): 453-456.

Kojima, K., R. Nishimura, T. Mutoh, S.H. Hong, M. Mochizuki and N. Sasaki (2002). Effects of medetomidine-midazolam, acepromazinebutorphanol, and midazolam-butorphanol on induction dose of thiopental and propofol and on cardiopulmonary changes in dogs. Am. J. Vet. Res. 63(12): 1671-1679.

Kandpal, M., N.S. Jadon, A. Kumar and S. Kumar (2005). Studies on reversal of clinicophysiological effects of xylazine with yohimbine in dogs. Indian J. Vet. Surg. 26(1): 39-40.

Le-Chevallier, D., L. Slingsby and J. Murrell (2019). Use of midazolam in combination with medetomidine for premedication in healthy dogs. Vet. Anaesth. Analg. 46(1): 74-78.

Mama, K.R., A.E. Wagner, E.P. Steffey, C. KolliasBaker, P.W. Hellyer, A.E. Golden and L.F. Brevard (2005). Evaluation of xylazine and ketamine for total intravenous anesthesia in horses. Am. J. Vet. Res. 66(6): 1002-1007.

Maravi, M.S., R. Dewangan, S.K. Tiwari, R. Sharda and M.O. Kalim (2018). Clinico-Physiological Response to Detomidine-Propofol Anaesthesia in Atropinized Goats. Int. J. Curr. Microbiol. Appl. Sci. 7(2): 2978-2983.

Mattos, J., A.H.H. Minervino, J. Barrêto, F.A.M.L. Rodrigues, C.A.S.C. Araújo, E.L. Ortolani and S.R.G. Cortopassi (2013). High doses of lidocaine as a constant rate infusion in propofol/fentanyl anaesthetized sheep: cardiorespiratory effects. Semin. Cienc. Agrar. 34(1): 323-334.

Mehlisch, D.R. (2002). The efficacy of combination analgesic therapy in relieving dental pain. J. Am. Dent. Assoc. 133(7): 861-871.

Njoku, N.U. (2015). Effects of maintenance of propofolketamine anesthesia with repeat bolus and constant rate infusion of propofol on physiological, biochemical, anesthetic and analgesic indices in dogs. J. Adv. Vet. Anim. Res. 2(4): 427-434.

Okwudili, C.U., A.E. Chinedu and J.O. Anayo (2014). Biochemical effects of xylazine, propofol, and ketamine in West African dwarf goats. J. Vet. Med. Article ID 758581.
Prassinos, N.N., A.D. Galatos and D. Raptopoulos (2005). A comparison of propofol, thiopental or ketamine as induction agents in goats. Vet. Anaesth. Analg. 32(5): 289-296.

Potliya, S., A. Kumar, S. Kumar, S. Singh and S. Kumar (2015). Evaluation of efficacy and safety of glycopyrrolate-xylazine-propofol anesthesia in buffalo calves. Vet. World. 8(3): 251-256.

Shah, Z., A.B. Kalhore, A.B. Kachiwal, I. Ahmad, H. Sattar, M.A. Khan and H.A. Ullah (2013). Comparative studies on sedative and analgesic effects of xylazine and detomidine in goats. J. Anim. Plant Sci. 23(1): 39-42.

Sinclair, M.D. (2003). A review of the physiological effects of $\alpha 2$-agonists related to the clinical use of medetomidine in small animal practice. Can. Vet. J. 44(11): 885.

Sahoo, M., I. Nath, S. Nayak, A.K. Kundu, S.K. Panda and B.K. Patra (2018). Comparision of sedative effect of dexmedetommine/xylazine in combination with butorphanol-midazolam as preanaesthetic to ketamine anaesthesia for ovariohysterectomy in dogs. Explor. Anim. Med. Res. 8(1): 79-84.

Tunio, A., S. Bughio, J.K. Sahito, M.G. Shah, M. Ebrahimi and S.P. Tunio (2016). Observation of Physiological Changes after Detomidine Administration in Pateri Goat. Mac. Vet. Rev. 39(1): 77-81

Ukwueze, C.O., C.A. Eze and R.I. Udegbunam (2014). Assessment of Common Anaesthetic and Clinical Indices of Multimodal Therapy of Propofol, Xylazine and Ketamine in Total Intravenous Anaesthesia in West African Dwarf Goat. J. Vet. Med. 1-14.

Waelbers, T., P. Vermoere and I. Polis (2009). Total intravenous anesthesia in dogs. Vlaams Diergeneeskundig Tijdschrift. 78(3): 160-169 p.

Wiese, A.J., P. Lerche, R.M. Cleale and W.W. Muir (2010). Investigation of escalating and large bolus doses of a novel, nano-droplet, aqueous $1 \%$ propofol formulation in cats. Vet. Anaesth. Analg. 37(3): 250-257.

Zeedan, K.I., O.M. El-Malky and A.A. Abu El-Ella (2014). Nutritional, Physiological and Microbiological Studies on Using Biogen-Zinc on Productive and Reproductive Performance of Ruminants: Productive Performance, Digestion and Some Blood Components of Damascus Goats. Egyptian J Sheep and Goat Sci. 65(1800): 1-36. 